\title{
MODEL FOR THE PREDICTION OF FLEXURAL STRENGHTS OF SAND STONE-PERIWINKLE SHELL CONCRETE
}

\author{
Ibearugbulem O.M. ${ }^{1}$, Ajoku C.A. ${ }^{2}$, Iwuoha S.E. ${ }^{3}$ \\ ${ }^{1}$ Senior Lecturer, Department of Civil Engineering, Federal University of Technology Owerri, Imo State, Nigeria \\ ${ }^{2}$ Assistant Lecturer, Department of Civil Engineering, Federal University of Technology Owerri, Imo State, Nigeria \\ ${ }^{3}$ Graduate Assistant, Department of Civil Engineering, Federal University of Technology Owerri, Imo State, Nigeria
}

\begin{abstract}
This research work presents the prediction of $28^{\text {th }}$ day flexural strength of lightweight concrete made from equal volume of periwinkle shells and sand stone. Regression equation for a four component mixture developed by Ibearugbulem was utilized. The materials used in the laboratory experiment include; water, Ordinary Portland Cement, river sand, sand stone, and periwinkle shells. Sand stone and periwinkle shells were blended to form a single component thereby reducing the number of material components from five to four. Thirty mix ratios obtained arbitrarily from Scheffe's simplex latex structure for a four component mixture were utilized. Batching of constituent materials was by weight except for the coarse aggregates (periwinkle shell and sand stone) which were batched by volume. Manual mixing operation was adopted in the laboratory. A total of ninety prototype concrete beams were produced from the given mix ratios. Whereas the first fifteen mix ratios termed the actual mix ratios was used for the formulation of the regression model, the last fifteen mix ratios regarded as the control mix ratios was used for the validation of the formulated model using statistical analysis. Fisher's statistical tool was utilized. From the analysis, the calculated value of fisher of 2.13 was less than the fisher value of 2.48 obtained from the statistical f-distribution table. This result proved that there was no much difference between the laboratory flexural strength values and the model flexural strength values at 5\% significance level. Therefore, this model is adequate for the accurate forecast of the $28^{\text {th }}$ day flexural strength of lightweight periwinkle shell-sand stone concrete when given mix ratios. It is therefore recommended for use in concretel construction industry.
\end{abstract}

Keywords: Flexural Strength, Periwinkle Shell-Sand Stone Concrete, Mix Ratio, Regression Model

\section{INTRODUCTION}

Concrete is one of the major building materials in civil engineering practice and construction works in Nigeria. Its basic constituents are cement, fine aggregates, coarse aggregate and water. The conventional normal weight coarse aggregates needed for construction purposes are expensive hence the need to source for suitable and more readily available alternative construction materials. These alternative coarse aggregates can be used wholly or partially to replace the expensive conventional normal weight coarse aggregate in the production of low cost housing unit for rural dwellers.

Periwinkle shell obtained from periwinkle, which is an available proteinous food to people living in riverine and coastal communities in Nigeria, has been investigated by several researchers as a coarse aggregate for lightweight concrete production. Adewuyi and Adegoke (2009), Amaziah et al. (2013), Awe et al. (2014), Elijah et al. (2009), Ettu et al. (2013), Falade et al. (2010), Njoku et al. (2011), Ohimain et al. (2009), and Osarenwinda and Awaro (2009) have all carried out series of investigations on the prospects of using periwinkle shells, which were regarded as pollutants due to their unsightly appearance in opendump sites as partial replacement for the unavailable and expensive conventional normal weight coarse aggregates in lightweight concrete production. These researchers having combined periwinkle shells at different partial replacements with the conventional normal weight coarse aggregate proved it to be adequate for lightweight concrete production. Another factor which could increase the cost of production of concrete is the enormous time and effort invested in carrying-out trial mixes for desired fresh or hardened concrete properties. To overcome this short coming, Ibearugbulem et al. (2013), Osadebe (2003) and Scheffe (1950) have all developed optimisation equations for the prediction of compressive and flexural strengths of concretes and sandcrete/lateritic blocks.

This research work therefore seeks to apply the regression equation developed by Ibearugbulem for a four component mixture to formulate a new model for the prediction of the $28^{\text {th }}$ day flexural strengths of periwinkle shell-river gravel concrete. The objective of this research if realised will help equip civil engineers working in concrete or construction industries with easy to use mathematical tool for predicting flexural strengths of lightweight concrete, thereby saving the enormous time and effort invested in carrying out trial mixes. It will also help manage the enormous pollution generated by periwinkle shell through their use as a construction material for lightweight concrete production. 


\subsection{Ibearugbulem's Regression Model}

Ibearugbulem et al. (2013) derived a regression equation for a four component mixture of concrete of degree four given in Equation 1. This equation forms the basis of the regression model developed in this research for the prediction of flexural strength of lightweight concrete having equal volume of periwinkle shells and river gravel.

$F(z)=\alpha_{1} z_{1}+\alpha_{2} z_{2}+\alpha_{3} z_{3}+\alpha_{4} z_{4}+\alpha_{12} z_{1} z_{2}+\alpha_{13} z_{1} z_{3}+\alpha_{14} z_{1} z_{4}$

$+\alpha_{23} Z_{2} z_{3}+\alpha_{24} z_{2} z_{4}+\alpha_{34} z_{3} z_{4}+\alpha_{123} Z_{1} z_{2} z_{3}+\alpha_{124} z_{1} z_{2} z_{4}+$

$\alpha_{134} Z_{1} Z_{3} Z_{4}+\alpha_{234} Z_{2} Z_{3} Z_{4}+\alpha_{1234} Z_{1} Z_{2} Z_{3} Z_{4}$

Where, $F(z)$ is the response function (i.e the property of concrete that is of interest).

$\alpha$ is the coefficient of the regression equation obtained from Equation 4.

$\mathrm{Z}$ is the pseudo variable obtained from Equations 2 and 3.

\subsection{Pseudo and Actual Variables}

The relationship between the pseudo variable, $\mathrm{z}_{\mathrm{i}}$ and actual variable, $\mathrm{s}_{\mathrm{i}}$ is as given below.

$\mathrm{Z}_{\mathrm{i}}=\mathrm{s}_{\mathrm{i}} / \mathrm{S}$

$\mathrm{S}=\sum \mathrm{S}_{\mathrm{i}}$

\subsection{Coefficient of the Regression Function}

The matrix expression below given by Ibearugbulem is used to obtain the coefficient of the regression function.

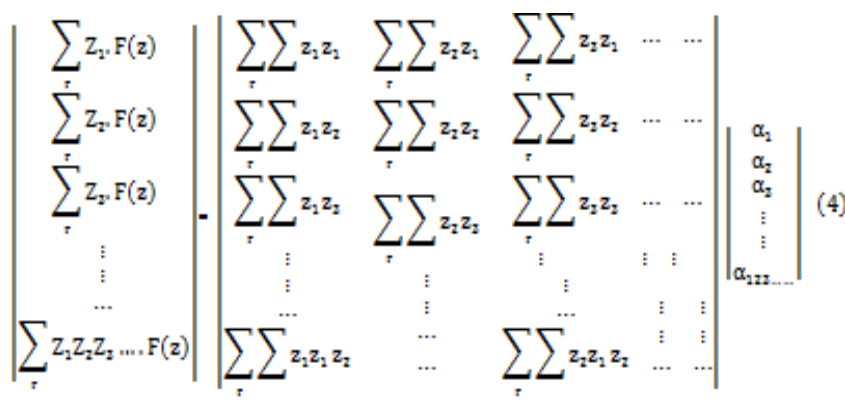

Equation 4 can be written in a short form as shown in Equation5.

$[\mathrm{F}(\mathrm{z}) \cdot \mathrm{Z}]=[\mathrm{CC}][\alpha]$

Where, $\mathrm{CC}$ is always a symmetric matrix. For a mixture of four components, CC is a $15 \times 15$ matrix shown in Table 2. Making $[\alpha]$ the subject of the equation gives Equation 6.

$[\propto]=[\mathrm{F}(\mathrm{z}) \cdot \mathrm{Z}] \cdot[C C]^{-1}$

\subsection{Hypothesis}

NULL HYPOTHESIS $\left(\mathrm{H}_{0}\right)$ : there is no significant difference between the results of the model and experimental test results.

ALTERNATIVE HYPOTHESIS $\left(\mathrm{H}_{1}\right)$ : there is a significant difference between the results of the model and the experimental test results.
The risk involved is that $5 \%$ or below of the results of the model will be incorrect.

\section{MATERIALS AND METHODS}

\subsection{Materials}

Five constituent materials were used in the laboratory to produce the prototype concrete beams measuring 150x150x600mm. These materials include Ordinary Portland cement, river sand, sand stone, periwinkle shell and water.

i. The Ordinary Portland cement with properties conforming to BS EN 197-1:2000 was used in the laboratory experiment.

ii. The river sand used was obtained from Otamiri River located in federal university of technology owerri. It has physical properties of $1670 \mathrm{~kg} / \mathrm{m}^{3}, 0.36,2.608$ and 2.52 corresponding to its values of un-compacted bulk density, void ratio, specific gravity and fineness modulus respectively. The river sand is uniformly graded because it has coefficient of uniformity and coefficient of curvature values of 2.32 and 0.87 respectively.

iii. The sand stone used in the experiment has a maximum size of $20 \mathrm{~mm}$ and irregular in shape. It has an uncompacted bulk density, water absorption, void ratio, specific gravity, fineness modulus, impact value and abrasion value of $1400 \mathrm{~kg} / \mathrm{m}^{3}, 2.3 \%, 0.41,2.358,4.08$, $23.88 \%$ and $42.00 \%$ respectively. The sand stone is poorly graded because of its values of coefficient of uniformity and curvature which are 1.18 and 0.92 respectively.

iv. The periwinkle shells which have a maximum size of $19 \mathrm{~mm}$ were obtained from Rumuagholu market in River state, Nigeria. It has physical and mechanical properties of $520 \mathrm{~kg} / \mathrm{m}^{3}, 2.4 \%, 0.55,1.16,3.72,33.50 \%$ and $55.04 \%$ which corresponds to the un-compacted bulk density, water absorption, void ratio, specific gravity, fineness modulus, impact value and abrasion value respectively. Its coefficient of uniformity and curvature values of 1.18 and 1.12 indicate a poorly graded particle size distribution.

v. Potable water was used for the experiment during mixing and curing operation.

\subsection{Methods}

\subsubsection{Concrete Materials Batching, Mixing, Placing}

\section{and Curing Operations}

Batching in this experiment was done with a weighing balance of $50 \mathrm{~kg}$ capacity using the mix ratios given in Table 1. These mix ratios were chosen arbitrarily from Scheffe's simplex latex structure for a four component mixture.

The batching for the different constituent materials was by mass except for the coarse aggregates (i.e sand stone and periwinkle shells) which were volumetrically combined at a mix ratio of $1: 1$. 
Manual mixing was adopted with the aid of spade. The constituent materials were mixed thoroughly until homogeneity was attained. After mixing, the concrete was cast into steel moulds measuring $150 \times 150 \times 600 \mathrm{~mm}$.

Three representative samples were cast for each mix ratio, making a total of 90 prototype concrete beams produced. They were cured for 28 days in an open curing tank filled with potable water. $100 \mathrm{kN}$ capacity hand operated flexural testing machined designed for third point loading was used to crush the cured concrete beams. Constant rate of loading was applied on the concrete beams until failure occurred. The laboratory modulus of rupture or flexural strength of each beam was determined using Equation 7. The results obtained are given on Table 3.

Flexural strength $=\frac{\text { load on beam } x \text { span }}{6 x \text { elastic modulus of beam }}$

\subsubsection{Goodness of Fit}

Fisher's test is used to test the adequacy of the model by comparing the properties (flexural strengths) of the "control samples" of the experiment with that of the model. The values obtained from the model is said to be adequate if $\frac{1}{f_{x}\left(v_{1}, v_{2}\right)}<\frac{s_{1}^{2}}{s_{2}^{2}}<f_{\alpha}\left(v_{1}, v_{2}\right)$.

Where,

$\frac{s_{1}^{2}}{s_{2}^{2}}=$ fisher value obtained from statistical analysis

$\mathrm{S}_{1}{ }^{2}=$ greater of $\mathrm{S}_{\mathrm{E}}{ }^{2}$ and $\mathrm{S}_{\mathrm{m}}{ }^{2}$

$\mathrm{S}_{\mathrm{m}}{ }^{2}=$ variance from the model

$\mathrm{S}_{\mathrm{E}}^{2}=$ variance from the experiment

$f_{\alpha}\left(v_{1}, v_{2}\right)=$ fisher value obtained from $F$ - distribution table. $\alpha=$ significant level $=5 \%=0.05$

$\mathrm{V}_{1}=\mathrm{V}_{2}=\mathrm{N}-1$ = degree of freedom.

Table 1: Actual Mix Ratios

\begin{tabular}{|c|c|c|c|c|c|}
\hline $\mathrm{S} / \mathrm{N}$ & MIX NO & WATER, $\mathrm{s}_{1}$ & CEMENT, $\mathrm{s}_{2}$ & $\mathrm{SAND}, \mathrm{s}_{3}$ & 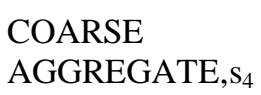 \\
\hline 1 & N1 & 0.6 & 1 & 2 & 4 \\
\hline 2 & $\mathrm{~N} 2$ & 0.55 & 1 & 2 & 3 \\
\hline 3 & N3 & 0.5 & 1 & 1.5 & 2 \\
\hline 4 & N4 & 0.45 & 1 & 1 & 1.5 \\
\hline 5 & N12 & 0.575 & 1 & 2 & 3.5 \\
\hline 6 & N13 & 0.55 & 1 & 1.75 & 3 \\
\hline 7 & N14 & 0.525 & 1 & 1.5 & 2.75 \\
\hline 8 & $\mathrm{~N} 23$ & 0.525 & 1 & 1.75 & 2.5 \\
\hline 9 & $\mathrm{~N} 24$ & 0.5 & 1 & 1.5 & 2.25 \\
\hline 10 & N34 & 0.475 & 1 & 1.25 & 1.75 \\
\hline 11 & N123 & 0.55 & 1 & 1.8333 & 3 \\
\hline 12 & N124 & 0.533333 & 1 & 1.6667 & 2.8333 \\
\hline 13 & N134 & 0.516667 & 1 & 1.5 & 2.5 \\
\hline 14 & N234 & 0.5 & 1 & 1.5 & 2.1667 \\
\hline 15 & N1234 & 0.525 & 1 & 1.675 & 2.625 \\
\hline 16 & $\mathrm{C} 1$ & 0.5175 & 1 & 1.575 & 2.475 \\
\hline 17 & $\mathrm{C} 2$ & 0.5275 & 1 & 1.675 & 2.65 \\
\hline 18 & C3 & 0.5375 & 1 & 1.7 & 2.85 \\
\hline 19 & $\mathrm{C} 4$ & 0.5175 & 1 & 1.55 & 2.525 \\
\hline 20 & $\mathrm{C5}$ & 0.521 & 1 & 1.575 & 2.565 \\
\hline 21 & C6 & 0.4985 & 1 & 1.485 & 2.16 \\
\hline 22 & $\mathrm{C7}$ & 0.5215 & 1 & 1.54 & 2.565 \\
\hline 23 & $\mathrm{C} 8$ & 0.5285 & 1 & 1.65 & 2.745 \\
\hline 24 & C9 & 0.5515 & 1 & 1.825 & 3.03 \\
\hline 25 & C10 & 0.5045 & 1 & 1.545 & 2.21 \\
\hline 26 & C11 & 0.585 & 1 & 2 & 3.7 \\
\hline 27 & $\mathrm{C} 12$ & 0.535 & 1 & 1.85 & 2.7 \\
\hline 28 & $\mathrm{C} 13$ & 0.485 & 1 & 1.35 & 1.85 \\
\hline 29 & C14 & 0.495 & 1 & 1.3 & 2.25 \\
\hline 30 & C15 & 0.5325 & 1 & 1.6625 & 2.65 \\
\hline 30 & C15 & 0.5325 & 1 & 1.6625 & 2.65 \\
\hline
\end{tabular}


Table 2: CC Matrix of a Mix of Four Components

\begin{tabular}{|c|c|c|c|c|c|c|c|c|c|c|c|c|c|c|}
\hline $\begin{array}{l}\Sigma \Sigma \mathrm{ZZ1} \\
\mathrm{Z1}\end{array}$ & $\begin{array}{l}\sum \sum \mathrm{Z1} \\
\mathrm{Z2}\end{array}$ & $\begin{array}{l}\Sigma \Sigma Z 1 \\
Z 3\end{array}$ & $\begin{array}{l}\sum \Sigma \mathrm{Z1} \\
\mathrm{Z4}\end{array}$ & $\begin{array}{l}\Sigma \sum Z 1 \\
Z 1 Z 2\end{array}$ & $\begin{array}{l}\Sigma \Sigma Z 1 \\
Z 1 Z 3\end{array}$ & $\begin{array}{l}\sum \Sigma Z 1 \\
Z 1 Z 4\end{array}$ & $\begin{array}{l}\Sigma \sum Z 1 \\
Z 2 Z 3\end{array}$ & $\begin{array}{l}\sum \sum Z 1 Z 2 \\
Z 4\end{array}$ & $\begin{array}{l}\sum \sum Z 1 \\
Z 3 Z 4\end{array}$ & $\begin{array}{l}\Sigma \Sigma Z 11 \\
Z 1 Z 2 \\
Z 3\end{array}$ & $\begin{array}{l}\sum \sum Z 1 Z 1 \\
Z 2 Z 4\end{array}$ & $\begin{array}{l}\sum \sum Z 1 Z 1 \\
Z 3 Z 4\end{array}$ & $\begin{array}{l}\sum \Sigma Z 1 Z 2 \\
Z 3 Z 4\end{array}$ & $\begin{array}{l}\sum \sum Z 1 Z 1 \\
Z 2 Z 3 Z 4\end{array}$ \\
\hline $\begin{array}{l}\Sigma \Sigma \mathrm{Z1} \\
\mathrm{Z2}\end{array}$ & $\begin{array}{l}\Sigma \Sigma \mathrm{ZZ} 2 \\
\mathrm{Z2}\end{array}$ & $\begin{array}{l}\Sigma \Sigma \mathrm{ZZ} \\
\mathrm{Z3}\end{array}$ & $\begin{array}{l}\Sigma \Sigma Z 2 \\
Z 4\end{array}$ & $\begin{array}{l}\Sigma \Sigma Z 1 \\
Z 2 Z 2\end{array}$ & $\begin{array}{l}\Sigma \Sigma Z 1 \\
Z 2 Z 3\end{array}$ & $\begin{array}{l}\Sigma \Sigma \mathrm{Z} 1 \\
\mathrm{Z} 2 \mathrm{Z4}\end{array}$ & $\begin{array}{l}\Sigma \Sigma Z 2 \\
Z 2 Z 3\end{array}$ & $\begin{array}{l}\sum \Sigma \mathrm{Z} 2 \mathrm{Z2} \\
\mathrm{Z4}\end{array}$ & $\begin{array}{l}\Sigma \Sigma Z 2 \\
Z 3 Z 4\end{array}$ & $\begin{array}{l}\sum \sum Z 1 \\
Z 2 Z 2 \\
Z 3\end{array}$ & $\begin{array}{l}\sum \sum Z 1 Z 2 \\
Z 2 Z 4\end{array}$ & $\begin{array}{l}\sum \Sigma \mathrm{Z1Z2} \\
\mathrm{Z} 3 \mathrm{Z} 4\end{array}$ & $\begin{array}{l}\sum \Sigma Z 2 Z 2 \\
Z 3 Z 4\end{array}$ & $\begin{array}{l}\Sigma \Sigma Z 1 Z 2 \\
Z 2 Z 3 Z 4\end{array}$ \\
\hline $\begin{array}{l}\Sigma \Sigma \mathrm{Z1} \\
\mathrm{Z3}\end{array}$ & $\begin{array}{l}\sum \sum Z 22 \\
Z 3\end{array}$ & $\begin{array}{l}\Sigma \Sigma Z 33 \\
Z 3\end{array}$ & $\begin{array}{l}\sum \Sigma Z 3 \\
Z 4\end{array}$ & $\begin{array}{l}\Sigma \Sigma Z 1 \\
Z 2 Z 3\end{array}$ & $\begin{array}{l}\Sigma \Sigma Z 1 \\
Z 3 Z 3\end{array}$ & $\begin{array}{l}\Sigma \Sigma Z 1 \\
Z 3 Z 4\end{array}$ & $\begin{array}{l}\Sigma \Sigma Z 2 \\
Z 3 Z 3\end{array}$ & $\begin{array}{l}\sum \Sigma \mathrm{ZZZ3} \\
\mathrm{Z4}\end{array}$ & $\begin{array}{l}\sum \sum Z 3 \\
Z 3 Z 4\end{array}$ & $\begin{array}{l}\Sigma \Sigma Z 1 \\
Z 2 Z 3 \\
Z 3\end{array}$ & $\begin{array}{l}\sum \sum Z 1 Z 2 \\
Z 3 Z 4\end{array}$ & $\begin{array}{l}\sum \Sigma \mathrm{Z1Z3} \\
\mathrm{Z} 3 \mathrm{Z4}\end{array}$ & $\begin{array}{l}\sum \Sigma \mathrm{Z} 2 \mathrm{Z3} \\
\mathrm{Z} Z \mathrm{Z4}\end{array}$ & $\begin{array}{l}\Sigma \Sigma Z 1 Z 2 \\
Z 3 Z 3 Z 4\end{array}$ \\
\hline $\begin{array}{l}\sum \Sigma \mathrm{Z1} \\
\mathrm{Z4}\end{array}$ & $\begin{array}{l}\Sigma \Sigma \mathrm{ZZ} 2 \\
\mathrm{Z4}\end{array}$ & $\begin{array}{l}\sum \Sigma Z 33 \\
Z 4\end{array}$ & $\begin{array}{l}\sum \Sigma \mathrm{Z4} \\
\mathrm{Z4}\end{array}$ & $\begin{array}{l}\Sigma \Sigma Z 1 \\
Z 2 Z 4\end{array}$ & $\begin{array}{l}\Sigma \Sigma Z 1 \\
Z 3 Z 4\end{array}$ & $\begin{array}{l}\Sigma \sum Z 1 \\
Z 4 Z 4\end{array}$ & $\begin{array}{l}\Sigma \Sigma Z 2 \\
Z 3 Z 4\end{array}$ & $\begin{array}{l}\sum \sum Z 2 Z 4 \\
Z 4\end{array}$ & $\begin{array}{l}\sum \sum Z 3 \\
Z 4 Z 4\end{array}$ & $\begin{array}{l}\Sigma \Sigma Z 1 \\
Z 2 Z 3 \\
Z 4\end{array}$ & $\begin{array}{l}\sum \sum Z 1 Z 2 \\
Z 4 Z 4\end{array}$ & $\begin{array}{l}\sum \sum Z 1 Z 3 \\
Z 4 Z 4\end{array}$ & $\begin{array}{l}\sum \sum Z 2 Z 3 \\
Z 4 Z 4\end{array}$ & $\begin{array}{l}\Sigma \Sigma Z 1 Z 2 \\
Z 3 Z 4 Z 4\end{array}$ \\
\hline $\begin{array}{l}\Sigma \Sigma Z 1 \\
Z 1 Z 2 \\
\end{array}$ & $\begin{array}{l}\Sigma \Sigma \mathrm{Z} 1 \\
\mathrm{Z} 2 \mathrm{Z2}\end{array}$ & $\begin{array}{l}\Sigma \Sigma Z 1 \\
Z 2 Z 3\end{array}$ & $\begin{array}{l}\Sigma \Sigma Z 1 \\
Z 2 Z 4\end{array}$ & $\begin{array}{l}\Sigma \sum Z 1 \\
\mathrm{Z} 1 \mathrm{Z2} \\
\mathrm{Z2}\end{array}$ & $\begin{array}{l}\Sigma \sum Z 11 \\
Z 1 Z 2 \\
Z 3\end{array}$ & $\begin{array}{l}\sum \sum Z 1 \\
Z 1 Z 2 \\
Z 4\end{array}$ & $\begin{array}{l}\Sigma \sum Z 1 \\
\mathrm{ZZZ2} \\
\mathrm{Z3}\end{array}$ & $\begin{array}{l}\Sigma \Sigma \mathrm{Z} 1 \mathrm{Z2} \\
\mathrm{Z} 2 \mathrm{Z4}\end{array}$ & $\begin{array}{l}\sum \sum Z 1 \\
Z 2 Z 3 \\
Z 4\end{array}$ & $\begin{array}{l}\Sigma \Sigma Z Z 1 \\
Z 1 Z 2 \\
Z 2 Z 3 \\
\end{array}$ & $\begin{array}{l}\Sigma \Sigma Z 1 Z 1 \\
Z 2 Z 2 Z 4\end{array}$ & $\begin{array}{l}\Sigma \Sigma Z 1 Z 1 \\
Z 2 Z 3 Z 4\end{array}$ & $\begin{array}{l}\Sigma \Sigma Z 1 Z 2 \\
Z 2 Z 3 Z 4\end{array}$ & $\begin{array}{l}\sum \sum Z 1 Z 1 \\
Z 2 Z 2 Z 3 \\
Z 4\end{array}$ \\
\hline $\begin{array}{l}\Sigma \Sigma Z 1 \\
Z 1 Z 3\end{array}$ & $\begin{array}{l}\Sigma \Sigma \mathrm{Z1} \\
\mathrm{Z} 2 \mathrm{Z3}\end{array}$ & $\begin{array}{l}\Sigma \Sigma Z 1 \\
Z 3 Z 3\end{array}$ & $\begin{array}{l}\Sigma \Sigma Z 1 \\
Z 3 Z 4\end{array}$ & $\begin{array}{l}\sum \sum Z 1 \\
Z 1 Z 2 \\
Z 3\end{array}$ & $\begin{array}{l}\Sigma \sum Z 1 \\
Z 1 Z 3 \\
Z 3\end{array}$ & $\begin{array}{l}\sum \sum Z 1 \\
Z 1 Z 3 \\
Z 4\end{array}$ & $\begin{array}{l}\Sigma \sum \mathrm{Z1} \\
\mathrm{Z} 2 \mathrm{Z3} \\
\mathrm{Z3}\end{array}$ & $\begin{array}{l}\sum \Sigma \mathrm{Z1Z2} \\
\mathrm{Z} 3 \mathrm{Z4}\end{array}$ & $\begin{array}{l}\sum \sum Z 1 \\
Z 3 Z 3 \\
Z 4\end{array}$ & $\begin{array}{l}\Sigma \Sigma Z 11 \\
Z 1 Z 2 \\
Z 3 Z 3\end{array}$ & $\begin{array}{l}\sum \sum Z 1 Z 1 \\
Z 2 Z 3 Z 4\end{array}$ & $\begin{array}{l}\Sigma \Sigma Z 1 Z 1 \\
Z 3 Z 3 Z 4\end{array}$ & $\begin{array}{l}\Sigma \Sigma Z 1 Z 2 \\
Z 3 Z 3 Z 4\end{array}$ & $\begin{array}{l}\sum \sum Z 1 Z 1 \\
Z 2 Z 3 Z 3 \\
Z 4\end{array}$ \\
\hline $\begin{array}{l}\sum \Sigma \mathrm{Z} 1 \\
\mathrm{Z} 1 \mathrm{Z4}\end{array}$ & $\begin{array}{l}\Sigma \Sigma Z 1 \\
Z 2 Z 4\end{array}$ & $\begin{array}{l}\Sigma \Sigma Z 1 \\
Z 3 Z 4\end{array}$ & $\begin{array}{l}\Sigma \Sigma Z 1 \\
Z 4 Z 4\end{array}$ & $\begin{array}{l}\Sigma \sum Z 1 \\
Z 1 Z 2 \\
Z 4\end{array}$ & $\begin{array}{l}\Sigma \sum Z 1 \\
Z 1 Z 3 \\
Z 4\end{array}$ & $\begin{array}{l}\sum \sum Z 1 \\
Z 1 Z 4 \\
Z 4\end{array}$ & $\begin{array}{l}\Sigma \Sigma \mathrm{Z1} \\
\mathrm{Z} Z \mathrm{Z3} \\
\mathrm{Z4}\end{array}$ & $\begin{array}{l}\sum \Sigma \mathrm{Z1Z2} \\
\mathrm{Z} 4 \mathrm{Z} 4\end{array}$ & $\begin{array}{l}\sum \sum Z 1 \\
Z 3 Z 4 \\
Z 4\end{array}$ & $\begin{array}{l}\Sigma \Sigma Z 1 \\
Z 1 Z 2 \\
Z 3 Z 4\end{array}$ & $\begin{array}{l}\sum \sum Z 1 Z 1 \\
Z 2 Z 4 Z 4\end{array}$ & $\begin{array}{l}\Sigma \Sigma Z 1 Z 1 \\
Z 3 Z 4 Z 4\end{array}$ & $\begin{array}{l}\Sigma \Sigma Z 1 Z 2 \\
Z 3 Z 4 Z 4\end{array}$ & $\begin{array}{l}\sum \sum Z 1 Z 1 \\
Z 2 Z 3 Z 4 \\
Z 4\end{array}$ \\
\hline $\begin{array}{l}\Sigma \Sigma \mathrm{Z} 1 \\
\mathrm{Z} 2 \mathrm{Z3}\end{array}$ & $\begin{array}{l}\Sigma \Sigma \mathrm{ZZ} \\
\mathrm{Z} 2 \mathrm{Z3}\end{array}$ & $\begin{array}{l}\Sigma \Sigma Z 2 \\
Z 3 Z 3\end{array}$ & $\begin{array}{l}\Sigma \Sigma Z 2 \\
Z 3 Z 4\end{array}$ & $\begin{array}{l}\Sigma \sum \mathrm{Z1} \\
\mathrm{Z} 2 \mathrm{Z2} \\
\mathrm{Z3}\end{array}$ & $\begin{array}{l}\Sigma \Sigma Z 1 \\
Z 2 Z 3 \\
Z 3\end{array}$ & $\begin{array}{l}\sum \sum Z 1 \\
Z 2 Z 3 \\
Z 4\end{array}$ & $\begin{array}{l}\Sigma \Sigma \mathrm{ZZ} \\
\mathrm{Z} 2 \mathrm{Z3} \\
\mathrm{Z3}\end{array}$ & $\begin{array}{l}\sum \Sigma Z 2 Z 2 \\
Z 3 Z 4\end{array}$ & $\begin{array}{l}\sum \sum Z 2 \\
Z 3 Z 3 \\
Z 4\end{array}$ & $\begin{array}{l}\Sigma \Sigma Z 11 \\
Z 2 Z 2 \\
Z 3 Z 3\end{array}$ & $\begin{array}{l}\Sigma \Sigma Z 1 Z 2 \\
Z 2 Z 3 Z Z 4\end{array}$ & $\begin{array}{l}\Sigma \Sigma Z 1 Z 2 \\
Z 3 Z 3 Z 4\end{array}$ & $\begin{array}{l}\Sigma \Sigma Z 2 Z 2 \\
Z 3 Z 3 Z 4\end{array}$ & $\begin{array}{l}\sum \sum Z 1 Z 2 \\
Z 2 Z 3 Z 3 \\
Z 4\end{array}$ \\
\hline $\begin{array}{l}\Sigma \Sigma Z 1 \\
Z 2 Z 4\end{array}$ & $\begin{array}{l}\Sigma \Sigma Z 2 \\
Z 2 Z 4\end{array}$ & $\begin{array}{l}\Sigma \Sigma Z 22 \\
Z 3 Z 4\end{array}$ & $\begin{array}{l}\Sigma \Sigma Z 2 \\
Z 4 Z 4\end{array}$ & $\begin{array}{l}\Sigma \Sigma Z 1 \\
Z 2 Z 2 \\
Z 4\end{array}$ & $\begin{array}{l}\Sigma \Sigma Z 11 \\
Z 2 Z 3 \\
Z 4\end{array}$ & $\begin{array}{l}\Sigma \Sigma Z 1 \\
Z 2 Z 4 \\
Z 4\end{array}$ & $\begin{array}{l}\Sigma \Sigma \mathrm{ZZ2} \\
\mathrm{Z} 2 \mathrm{Z3} \\
\mathrm{Z4}\end{array}$ & $\begin{array}{l}\Sigma \Sigma Z 2 Z 2 \\
Z 4 Z 4\end{array}$ & $\begin{array}{l}\sum \sum Z 2 \\
Z 3 Z 4 \\
Z 4 \\
\end{array}$ & $\begin{array}{l}\Sigma \Sigma Z Z 1 \\
Z 2 Z 2 \\
Z 3 Z 4\end{array}$ & $\begin{array}{l}\Sigma \Sigma Z 1 Z 2 \\
Z 2 Z 4 Z 4\end{array}$ & $\begin{array}{l}\Sigma \Sigma Z 1 Z 2 \\
Z 3 Z 4 Z 4\end{array}$ & $\begin{array}{l}\Sigma \Sigma Z 2 Z 2 \\
Z 3 Z 4 Z 4\end{array}$ & $\begin{array}{l}\Sigma \Sigma Z 1 Z 2 \\
Z 2 Z 3 Z 4 \\
Z 4\end{array}$ \\
\hline $\begin{array}{l}\Sigma \Sigma Z 1 \\
Z 3 Z 4\end{array}$ & $\begin{array}{l}\Sigma \Sigma Z 2 \\
Z 3 Z 4\end{array}$ & $\begin{array}{l}\Sigma \Sigma Z 3 \\
Z 3 Z 4\end{array}$ & $\begin{array}{l}\Sigma \Sigma Z 3 \\
Z 4 Z 4\end{array}$ & $\begin{array}{l}\sum \sum Z 1 \\
Z 2 Z 3 \\
Z 4\end{array}$ & $\begin{array}{l}\sum \sum Z 1 \\
Z 3 Z 3 \\
Z 4\end{array}$ & $\begin{array}{l}\Sigma \sum Z 1 \\
Z 3 Z 4 \\
Z 4\end{array}$ & $\begin{array}{l}\Sigma \Sigma Z 2 \\
Z 3 Z 3 \\
Z 4\end{array}$ & $\begin{array}{l}\sum \sum Z 2 Z 3 \\
Z 4 Z 4\end{array}$ & $\begin{array}{l}\Sigma \sum Z 3 \\
Z 3 Z 4 \\
Z 4\end{array}$ & $\begin{array}{l}\Sigma \Sigma Z Z 1 \\
Z 2 Z 3 \\
Z 3 Z 4\end{array}$ & $\begin{array}{l}\Sigma \Sigma Z 1 Z 2 \\
Z 3 Z 4 Z 4\end{array}$ & $\begin{array}{l}\Sigma \Sigma Z 1 Z 3 \\
Z 3 Z 4 Z 4\end{array}$ & $\begin{array}{l}\Sigma \Sigma \mathrm{ZZZZ} \\
\mathrm{Z} 3 \mathrm{Z} 4 \mathrm{Z4}\end{array}$ & $\begin{array}{l}\sum \sum Z 1 Z 2 \\
Z 3 Z 3 Z 4 \\
Z 4\end{array}$ \\
\hline $\begin{array}{l}\Sigma \Sigma Z 1 \\
\mathrm{Z} 1 \mathrm{Z2} \\
\mathrm{Z3}\end{array}$ & $\begin{array}{l}\Sigma \Sigma Z 1 \\
Z 2 Z 2 \\
Z 3\end{array}$ & $\begin{array}{l}\Sigma \Sigma \mathrm{Z} 1 \\
Z 2 Z 3 \\
Z 3\end{array}$ & $\begin{array}{l}\Sigma \Sigma Z 1 \\
Z 2 Z 3 \\
Z 4\end{array}$ & $\begin{array}{l}\Sigma \Sigma Z 1 \\
Z 1 Z 2 \\
Z 2 Z 3\end{array}$ & $\begin{array}{l}\Sigma \Sigma Z 1 \\
Z 1 Z 2 \\
Z 3 Z 3\end{array}$ & $\begin{array}{l}\Sigma \Sigma Z 1 \\
Z 1 Z 2 \\
Z 3 Z 4\end{array}$ & $\begin{array}{l}\Sigma \Sigma Z 1 \\
Z 2 Z 2 \\
Z 3 Z 3\end{array}$ & $\begin{array}{l}\sum \Sigma \mathrm{Z} 1 \mathrm{Z2} \\
\mathrm{Z} Z \mathrm{ZZZ4}\end{array}$ & $\begin{array}{l}\Sigma \Sigma Z 1 \\
Z 2 Z 3 \\
Z 3 Z 4\end{array}$ & $\begin{array}{l}\Sigma \Sigma Z 11 \\
Z 1 Z 2 \\
Z 2 Z 3 \\
Z 3\end{array}$ & $\begin{array}{l}\Sigma \Sigma Z 1 Z 1 \\
Z 2 Z 2 Z 3 \\
Z 4\end{array}$ & $\begin{array}{l}\Sigma \Sigma Z 1 Z 1 \\
Z 2 Z 3 Z 3 \\
Z 4\end{array}$ & $\begin{array}{l}\Sigma \Sigma Z 1 Z 2 \\
Z 2 Z 3 Z 3 \\
Z 4\end{array}$ & $\begin{array}{l}\Sigma \Sigma Z 1 Z 1 \\
Z 2 Z 2 Z 3 \\
Z 3 Z 4\end{array}$ \\
\hline $\begin{array}{l}\Sigma \Sigma Z 1 \\
Z 1 Z 2 \\
Z 4\end{array}$ & $\begin{array}{l}\Sigma \Sigma Z 1 \\
Z 2 Z 2 \\
Z 4\end{array}$ & $\begin{array}{l}\Sigma \Sigma Z 1 \\
Z 2 Z 3 \\
Z 4\end{array}$ & $\begin{array}{l}\Sigma \Sigma Z 1 \\
Z 2 Z 4 \\
Z 4\end{array}$ & $\begin{array}{l}\Sigma \Sigma Z 1 \\
Z 1 Z 2 \\
Z 2 Z 4\end{array}$ & $\begin{array}{l}\Sigma \Sigma Z 1 \\
Z 1 Z 2 \\
Z 3 Z 4\end{array}$ & $\begin{array}{l}\Sigma \Sigma \mathrm{Z} 1 \\
Z 1 Z 2 \\
Z 4 Z 4\end{array}$ & $\begin{array}{l}\Sigma \Sigma Z 1 \\
Z 2 Z 2 \\
Z 3 Z 4\end{array}$ & $\begin{array}{l}\sum \Sigma \mathrm{Z} 1 \mathrm{Z2} \\
\mathrm{Z} Z \mathrm{Z} 4 \mathrm{Z4}\end{array}$ & $\begin{array}{l}\Sigma \Sigma \mathrm{Z1} \\
Z 2 Z 3 \\
Z 4 Z 4\end{array}$ & $\begin{array}{l}\Sigma \Sigma Z 1 \\
Z 1 Z 2 \\
Z 2 Z 3 \\
Z 4\end{array}$ & $\begin{array}{l}\Sigma \Sigma Z 1 Z 1 \\
Z 2 Z 2 Z 4 \\
Z 4\end{array}$ & $\begin{array}{l}\Sigma \Sigma Z 1 Z 1 \\
Z 2 Z 3 Z 4 \\
Z 4\end{array}$ & $\begin{array}{l}\Sigma \Sigma Z 1 Z 2 \\
Z 2 Z 3 Z 4 \\
Z 4\end{array}$ & $\begin{array}{l}\Sigma \Sigma \mathrm{Z} 1 \mathrm{Z1} \\
\mathrm{Z} Z \mathrm{ZZZ3} \\
\mathrm{Z} Z \mathrm{Z4}\end{array}$ \\
\hline $\begin{array}{l}\Sigma \Sigma Z 1 \\
Z 1 Z 3 \\
Z 4\end{array}$ & $\begin{array}{l}\Sigma \Sigma Z 1 \\
Z 2 Z 3 \\
Z 4\end{array}$ & $\begin{array}{l}\Sigma \Sigma Z 1 \\
Z 3 Z 3 \\
Z 4\end{array}$ & $\begin{array}{l}\Sigma \Sigma Z 1 \\
Z 3 Z 4 \\
Z 4\end{array}$ & $\begin{array}{l}\Sigma \Sigma Z 1 \\
Z 1 Z 2 \\
Z 3 Z 4\end{array}$ & $\begin{array}{l}\Sigma \Sigma Z 1 \\
Z 1 Z 3 \\
Z 3 Z 4\end{array}$ & $\begin{array}{l}\Sigma \Sigma Z 1 \\
Z 1 Z 3 \\
Z 4 Z 4\end{array}$ & $\begin{array}{l}\Sigma \Sigma Z 1 \\
Z 2 Z 3 \\
Z 3 Z 4\end{array}$ & $\begin{array}{l}\Sigma \Sigma Z 1 Z 2 \\
Z 3 Z 4 Z 4\end{array}$ & $\begin{array}{l}\Sigma \Sigma Z 1 \\
Z 3 Z 3 \\
Z 4 Z 4\end{array}$ & $\begin{array}{l}\Sigma \Sigma Z 1 \\
Z 1 Z 2 \\
Z 3 Z 3 \\
Z 4\end{array}$ & $\begin{array}{l}\Sigma \Sigma Z 1 Z 1 \\
Z 2 Z 3 Z 4 \\
Z 4\end{array}$ & $\begin{array}{l}\sum \sum Z 1 Z 1 \\
Z 3 Z 3 Z 4 \\
Z 4\end{array}$ & $\begin{array}{l}\Sigma \Sigma Z 1 Z 2 \\
Z 3 Z 3 Z 4 \\
Z 4\end{array}$ & $\begin{array}{l}\sum \Sigma Z 1 Z 1 \\
Z 2 Z 3 Z 3 \\
Z 4 Z 4\end{array}$ \\
\hline $\begin{array}{l}\Sigma \Sigma Z 1 \\
Z 2 Z 3 \\
Z 4\end{array}$ & $\begin{array}{l}\Sigma \Sigma Z 2 \\
Z 2 Z 3 \\
Z 4\end{array}$ & $\begin{array}{l}\Sigma \Sigma Z 2 \\
Z 3 Z 3 \\
Z 4\end{array}$ & $\begin{array}{l}\Sigma \Sigma Z 2 \\
Z 3 Z 4 \\
Z 4\end{array}$ & $\begin{array}{l}\Sigma \Sigma Z 1 \\
Z 2 Z 2 \\
Z 3 Z 4\end{array}$ & $\begin{array}{l}\Sigma \Sigma Z 1 \\
Z 2 Z 3 \\
Z 3 Z 4\end{array}$ & $\begin{array}{l}\Sigma \Sigma Z 1 \\
Z 2 Z 3 \\
Z 4 Z 4\end{array}$ & $\begin{array}{l}\Sigma \Sigma Z 2 \\
Z 2 Z 3 \\
Z 3 Z 4\end{array}$ & $\begin{array}{l}\Sigma \Sigma Z 2 Z 2 \\
Z 3 Z 3 Z 4\end{array}$ & $\begin{array}{l}\Sigma \Sigma Z 2 \\
Z 3 Z 3 \\
Z 4 Z 4\end{array}$ & $\begin{array}{l}\Sigma \Sigma Z 1 \\
Z 2 Z 2 \\
Z 3 Z 3 \\
Z 4\end{array}$ & $\begin{array}{l}\Sigma \Sigma Z 1 Z 2 \\
Z 2 Z 3 Z 4 \\
Z 4\end{array}$ & $\begin{array}{l}\Sigma \Sigma Z 1 Z 2 \\
Z 3 Z 3 Z 4 \\
Z 4\end{array}$ & $\begin{array}{l}\Sigma \Sigma Z 2 Z 2 \\
Z 3 Z 3 Z 4 \\
Z 4\end{array}$ & $\begin{array}{l}\Sigma \Sigma Z 1 Z 2 \\
Z 2 Z 3 Z 3 \\
Z 4 Z 4\end{array}$ \\
\hline $\begin{array}{l}\Sigma \Sigma Z 1 \\
Z 1 Z 2 \\
Z 3 Z 4\end{array}$ & $\begin{array}{l}\Sigma \Sigma Z 1 \\
Z 2 Z 2 \\
Z 3 Z 4\end{array}$ & $\begin{array}{l}\Sigma \Sigma Z 1 \\
Z 2 Z 3 \\
Z 3 Z 4\end{array}$ & $\begin{array}{l}\Sigma \Sigma Z 1 \\
Z 2 Z 3 \\
Z 4 Z 4\end{array}$ & $\begin{array}{l}\Sigma \sum Z 1 \\
Z 1 Z 2 \\
Z 2 Z 3 \\
Z 4\end{array}$ & $\begin{array}{l}\Sigma \sum Z 1 \\
Z 1 Z 2 \\
Z 3 Z 3 \\
Z 4\end{array}$ & $\begin{array}{l}\Sigma \Sigma Z 1 \\
Z 1 Z 2 \\
Z 3 Z 4 \\
Z 4\end{array}$ & $\begin{array}{l}\Sigma \sum Z 1 \\
Z 2 Z 2 \\
Z 3 Z 3 \\
\text { Z4 }\end{array}$ & $\begin{array}{l}\Sigma \Sigma Z 1 Z 2 \\
Z 2 Z 3 Z 3 \\
Z 4\end{array}$ & $\begin{array}{l}\Sigma \Sigma Z 1 \\
Z 2 Z 3 \\
Z 3 Z 4 \\
Z 4\end{array}$ & $\begin{array}{l}\Sigma \Sigma Z 1 \\
Z 1 Z 2 \\
Z 2 Z 3 \\
Z 3 Z 4\end{array}$ & $\begin{array}{l}\Sigma \Sigma Z 1 Z 1 \\
Z 2 Z 2 Z 3 \\
Z 4 Z 4\end{array}$ & $\begin{array}{l}\Sigma \Sigma Z 1 Z 1 \\
Z 2 Z 3 Z 3 \\
Z 4 Z 4\end{array}$ & $\begin{array}{l}\Sigma \Sigma \mathrm{Z} 1 \mathrm{Z2} \\
\mathrm{Z} Z \mathrm{Z} 3 \mathrm{Z3} \\
\mathrm{Z} \mathrm{ZZ}\end{array}$ & $\begin{array}{l}\Sigma \sum Z 1 Z 1 \\
Z 2 Z 2 Z 3 \\
Z 3 Z 4 Z 4\end{array}$ \\
\hline
\end{tabular}

\section{RESULTS AND DISCUSSION}

\subsection{Grain Size Distribution of River Sand, Sand}

\section{Stone and Periwinkle Shells}

Figures 1, 2 and 3 represent the grain size distribution of river sand, sand stone and periwinkle shells used in the laboratory experiment.

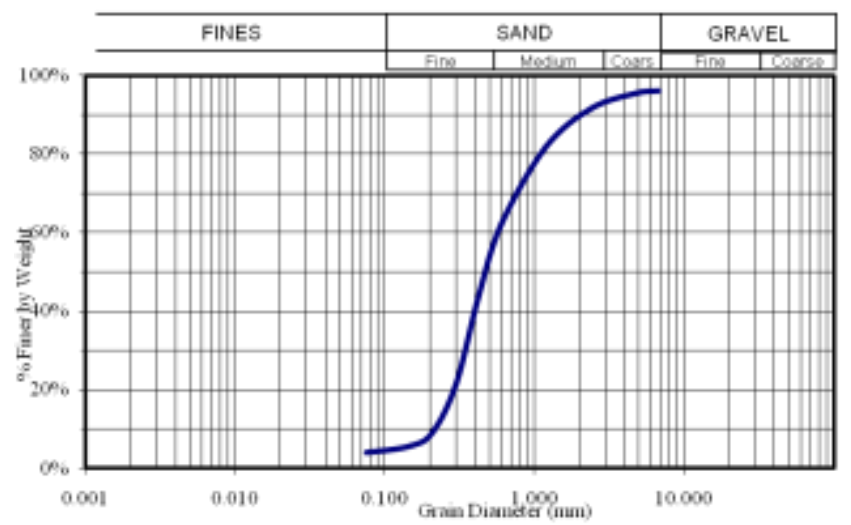

Figure 1: Grain size distribution analysis for river sand 


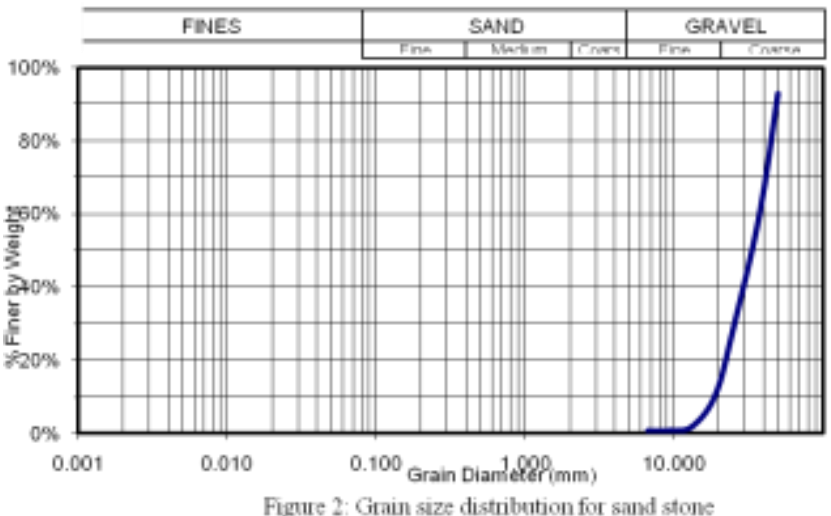

Figure 2: Grain size distribution for sand stone

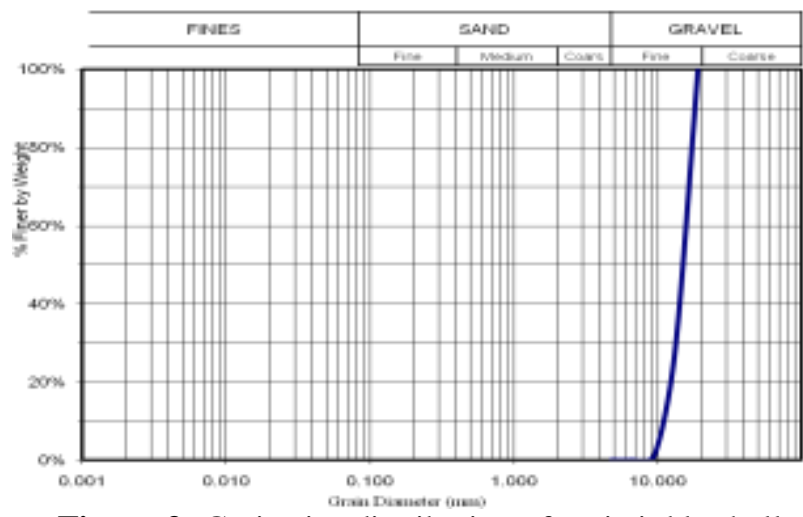

Figure 3: Grain size distribution of periwinkle shells

\subsection{Laboratory Flexural Strengths}

Table 3: Laboratory Flexural Strengths of Periwinkle shell-sand stone concrete

\begin{tabular}{|c|c|c|c|c|c|c|c|c|c|c|c|c|c|c|c|}
\hline \multicolumn{16}{|c|}{ ACTUAL MIX RATIOS } \\
\hline MIX NO & N1 & $\mathrm{N} 2$ & N3 & $\mathrm{N} 4$ & $\mathrm{~N} 12$ & N13 & N14 & $\mathrm{N} 23$ & $\mathrm{~N} 24$ & $\mathrm{~N} 34$ & $\mathrm{~N} 123$ & $\mathrm{~N} 124$ & N134 & $\mathrm{N} 234$ & N1234 \\
\hline $\begin{array}{l}\text { AVERAGE } \\
\text { FLEXURAL } \\
\text { STRENGTH } \\
\left(\mathrm{N} / \mathrm{mm}^{2}\right)\end{array}$ & 2.63 & 2.69 & 2.49 & 3.13 & 3.12 & 3.29 & 2.4 & 2.77 & 2.67 & 3.04 & 3.17 & 2.65 & 3.54 & 3.04 & 3.15 \\
\hline \multicolumn{16}{|c|}{ CONTROL MIX RATIOS } \\
\hline MIX NO & $\mathrm{C} 1$ & $\mathrm{C} 2$ & $\mathrm{C} 3$ & $\mathrm{C} 4$ & $\mathrm{C} 5$ & C6 & C7 & $\mathrm{C} 8$ & C9 & $\mathrm{C} 10$ & C11 & $\mathrm{C} 12$ & $\mathrm{C} 13$ & $\mathrm{C} 14$ & $\mathrm{C} 15$ \\
\hline $\begin{array}{l}\text { AVERAGE } \\
\text { FLEXURAL } \\
\text { STRENGTH } \\
\left(\mathrm{N} / \mathrm{mm}^{2}\right)\end{array}$ & 2.52 & 2.45 & 2.52 & 2.85 & 2.77 & 3.05 & 2.97 & 2.75 & 2.87 & 2.94 & 2.34 & 1.9 & 2.67 & 2.79 & 2.58 \\
\hline
\end{tabular}

\subsection{Flexural Strength From Formulated Regression}

\section{Model}

\subsection{1pseudo Variables, $Z_{i}$}

\begin{tabular}{|c|c|c|c|c|c|c|c|c|c|c|c|c|c|c|}
\hline \multicolumn{15}{|c|}{ Table 4: Pseudo Variables $\left(Z_{i}\right)$} \\
\hline $\mathrm{Z} 1$ & $\mathrm{Z} 2$ & $\mathrm{Z3}$ & $\mathrm{Z} 4$ & Z1Z2 & Z1Z3 & Z1Z4 & Z2Z3 & Z2Z4 & Z3Z4 & $\begin{array}{l}\text { Z1Z2Z } \\
3\end{array}$ & $\begin{array}{l}\text { Z1Z2Z } \\
4\end{array}$ & $\begin{array}{l}\text { Z1Z3Z } \\
4\end{array}$ & $\begin{array}{l}\text { Z2Z3Z } \\
4\end{array}$ & $\begin{array}{l}\text { Z1Z2Z } \\
3 Z 4\end{array}$ \\
\hline 0.079 & 0.132 & 0.263 & 0.526 & 0.010 & 0.021 & 0.042 & 0.035 & 0.069 & 0.139 & 0.003 & 0.005 & 0.011 & 0.018 & 0.001 \\
\hline 0.084 & 0.153 & 0.305 & 0.458 & 0.013 & 0.026 & 0.038 & 0.047 & 0.070 & 0.140 & 0.004 & 0.006 & 0.012 & 0.021 & 0.002 \\
\hline 0.100 & 0.200 & 0.300 & 0.400 & 0.020 & 0.030 & 0.040 & 0.060 & 0.080 & 0.120 & 0.006 & 0.008 & 0.012 & 0.024 & 0.002 \\
\hline 0.114 & 0.253 & 0.253 & 0.380 & 0.029 & 0.029 & 0.043 & 0.064 & 0.096 & 0.096 & 0.007 & 0.011 & 0.011 & 0.024 & 0.003 \\
\hline 0.081 & 0.141 & 0.283 & 0.495 & 0.011 & 0.023 & 0.040 & 0.040 & 0.070 & 0.140 & 0.003 & 0.006 & 0.011 & 0.020 & 0.002 \\
\hline 0.087 & 0.159 & 0.278 & 0.476 & 0.014 & 0.024 & 0.042 & 0.044 & 0.076 & 0.132 & 0.004 & 0.007 & 0.012 & 0.021 & 0.002 \\
\hline 0.091 & 0.173 & 0.260 & 0.476 & 0.016 & 0.024 & 0.043 & 0.045 & 0.082 & 0.124 & 0.004 & 0.008 & 0.011 & 0.021 & 0.002 \\
\hline 0.091 & 0.173 & 0.303 & 0.433 & 0.016 & 0.028 & 0.039 & 0.052 & 0.075 & 0.131 & 0.005 & 0.007 & 0.012 & 0.023 & 0.002 \\
\hline 0.095 & 0.190 & 0.286 & 0.429 & 0.018 & 0.027 & 0.041 & 0.054 & 0.082 & 0.122 & 0.005 & 0.008 & 0.012 & 0.023 & 0.002 \\
\hline 0.106 & 0.223 & 0.279 & 0.391 & 0.024 & 0.030 & 0.042 & 0.062 & 0.087 & 0.109 & 0.007 & 0.009 & 0.012 & 0.024 & 0.003 \\
\hline 0.086 & 0.157 & 0.287 & 0.470 & 0.013 & 0.025 & 0.040 & 0.045 & 0.074 & 0.135 & 0.004 & 0.006 & 0.012 & 0.021 & 0.002 \\
\hline 0.088 & 0.166 & 0.276 & 0.470 & 0.015 & 0.024 & 0.042 & 0.046 & 0.078 & 0.130 & 0.004 & 0.007 & 0.011 & 0.022 & 0.002 \\
\hline 0.094 & 0.181 & 0.272 & 0.453 & 0.017 & 0.025 & 0.042 & 0.049 & 0.082 & 0.123 & 0.005 & 0.008 & 0.012 & 0.022 & 0.002 \\
\hline 0.097 & 0.194 & 0.290 & 0.419 & 0.019 & 0.028 & 0.041 & 0.056 & 0.081 & 0.122 & 0.005 & 0.008 & 0.012 & 0.024 & 0.002 \\
\hline 0.091 & 0.173 & 0.281 & 0.455 & 0.016 & 0.026 & 0.041 & 0.049 & 0.079 & 0.128 & 0.004 & 0.007 & 0.012 & 0.022 & 0.002 \\
\hline
\end{tabular}

The pseudo variable values given in Table 4 are obtained using Table 1 and Equations 2 and 3.

\subsubsection{MATRIX}




\begin{tabular}{|c|c|c|c|c|c|c|c|c|c|c|c|c|c|c|}
\hline 0.093 & 0.180 & 0.283 & 0.445 & 0.017 & 0.026 & 0.041 & 0.051 & 0.080 & 0.126 & 0.005 & 0.007 & 0.012 & 0.023 & 0.002 \\
\hline 0.090 & 0.171 & 0.286 & 0.453 & 0.015 & 0.026 & 0.041 & 0.049 & 0.077 & 0.130 & 0.004 & 0.007 & 0.012 & 0.022 & 0.002 \\
\hline 0.088 & 0.164 & 0.279 & 0.468 & 0.015 & 0.025 & 0.041 & 0.046 & 0.077 & 0.131 & 0.004 & 0.007 & 0.012 & 0.021 & 0.002 \\
\hline 0.093 & 0.179 & 0.277 & 0.452 & 0.017 & 0.026 & 0.042 & 0.050 & 0.081 & 0.125 & 0.005 & 0.007 & 0.012 & 0.022 & 0.002 \\
\hline 0.092 & 0.177 & 0.278 & 0.453 & 0.016 & 0.026 & 0.042 & 0.049 & 0.080 & 0.126 & 0.005 & 0.007 & 0.012 & 0.022 & 0.002 \\
\hline 0.097 & 0.194 & 0.289 & 0.420 & 0.019 & 0.028 & 0.041 & 0.056 & 0.082 & 0.121 & 0.005 & 0.008 & 0.012 & 0.024 & 0.002 \\
\hline 0.093 & 0.178 & 0.274 & 0.456 & 0.016 & 0.025 & 0.042 & 0.049 & 0.081 & 0.125 & 0.005 & 0.008 & 0.012 & 0.022 & 0.002 \\
\hline 0.089 & 0.169 & 0.279 & 0.463 & 0.015 & 0.025 & 0.041 & 0.047 & 0.078 & 0.129 & 0.004 & 0.007 & 0.012 & 0.022 & 0.002 \\
\hline 0.086 & 0.156 & 0.285 & 0.473 & 0.013 & 0.025 & 0.041 & 0.044 & 0.074 & 0.135 & 0.004 & 0.006 & 0.012 & 0.021 & 0.002 \\
\hline 0.096 & 0.190 & 0.294 & 0.420 & 0.018 & 0.028 & 0.040 & 0.056 & 0.080 & 0.123 & 0.005 & 0.008 & 0.012 & 0.023 & 0.002 \\
\hline 0.080 & 0.137 & 0.275 & 0.508 & 0.011 & 0.022 & 0.041 & 0.038 & 0.070 & 0.139 & 0.003 & 0.006 & 0.011 & 0.019 & 0.002 \\
\hline 0.088 & 0.164 & 0.304 & 0.444 & 0.014 & 0.027 & 0.039 & 0.050 & 0.073 & 0.135 & 0.004 & 0.006 & 0.012 & 0.022 & 0.002 \\
\hline 0.104 & 0.213 & 0.288 & 0.395 & 0.022 & 0.030 & 0.041 & 0.062 & 0.084 & 0.114 & 0.006 & 0.009 & 0.012 & 0.024 & 0.003 \\
\hline 0.098 & 0.198 & 0.258 & 0.446 & 0.019 & 0.025 & 0.044 & 0.051 & 0.088 & 0.115 & 0.005 & 0.009 & 0.011 & 0.023 & 0.002 \\
\hline 0.091 & 0.171 & 0.284 & 0.453 & 0.016 & 0.026 & 0.041 & 0.049 & 0.078 & 0.129 & 0.004 & 0.007 & 0.012 & 0.022 & 0.002 \\
\hline
\end{tabular}

Substituting the values of Table 4 into Table 2, the numeric values of CC matrix is obtained as given in Table 5. The inverse of the matrix (Table 5) is given in Table 6.

\begin{tabular}{|c|c|c|c|c|c|c|c|c|c|c|c|c|c|c|}
\hline \multicolumn{15}{|c|}{ Table 5: CC Matrix } \\
\hline 0.129 & 0.250 & 0.389 & 0.616 & 0.024 & 0.036 & 0.057 & 0.070 & 0.110 & 0.173 & 0.007 & 0.010 & 0.016 & 0.031 & 0.003 \\
\hline 0.129 & 0.488 & 0.749 & 1.181 & 0.047 & 0.070 & 0.110 & 0.137 & 0.213 & 0.331 & 0.013 & 0.020 & 0.031 & 0.060 & 0.006 \\
\hline 0.389 & 0.749 & 1.189 & 1.891 & 0.070 & 0.109 & 0.173 & 0.211 & 0.331 & 0.533 & 0.020 & 0.031 & 0.049 & 0.093 & 0.009 \\
\hline 0.616 & 1.181 & 1.891 & 3.042 & 0.110 & 0.173 & 0.277 & 0.331 & 0.527 & 0.854 & 0.031 & 0.049 & 0.078 & 0.148 & 0.014 \\
\hline 0.024 & 0.047 & 0.070 & 0.110 & 0.004 & 0.007 & 0.010 & 0.013 & 0.020 & 0.031 & 0.001 & 0.002 & 0.003 & 0.006 & 0.001 \\
\hline 0.036 & 0.070 & 0.110 & 0.173 & 0.007 & 0.010 & 0.016 & 0.020 & 0.031 & 0.049 & 0.002 & 0.003 & 0.004 & 0.009 & 0.001 \\
\hline 0.057 & 0.110 & 0.173 & 0.277 & 0.010 & 0.016 & 0.025 & 0.031 & 0.049 & 0.078 & 0.003 & 0.005 & 0.007 & 0.014 & 0.001 \\
\hline 0.070 & 0.137 & 0.211 & 0.331 & 0.013 & 0.020 & 0.031 & 0.038 & 0.060 & 0.093 & 0.004 & 0.006 & 0.009 & 0.017 & 0.002 \\
\hline 0.110 & 0.213 & 0.331 & 0.527 & 0.020 & 0.031 & 0.049 & 0.060 & 0.094 & 0.148 & 0.006 & 0.009 & 0.014 & 0.026 & 0.002 \\
\hline 0.173 & 0.331 & 0.533 & 0.854 & 0.031 & 0.049 & 0.078 & 0.093 & 0.148 & 0.240 & 0.009 & 0.014 & 0.022 & 0.042 & 0.004 \\
\hline 0.007 & 0.013 & 0.020 & 0.031 & 0.001 & 0.002 & 0.003 & 0.004 & 0.006 & 0.009 & 0.000 & 0.001 & 0.001 & 0.002 & 0.000 \\
\hline 0.010 & 0.020 & 0.031 & 0.049 & 0.002 & 0.003 & 0.005 & 0.006 & 0.009 & 0.014 & 0.001 & 0.001 & 0.001 & 0.002 & 0.000 \\
\hline 0.016 & 0.031 & 0.049 & 0.078 & 0.003 & 0.004 & 0.007 & 0.009 & 0.014 & 0.022 & 0.001 & 0.001 & 0.002 & 0.004 & 0.000 \\
\hline 0.031 & 0.060 & 0.093 & 0.148 & 0.006 & 0.009 & 0.014 & 0.017 & 0.026 & 0.042 & 0.002 & 0.002 & 0.004 & 0.007 & 0.001 \\
\hline 0.003 & 0.006 & 0.009 & 0.014 & 0.001 & 0.001 & 0.001 & 0.002 & 0.002 & 0.004 & 0.000 & 0.000 & 0.000 & 0.001 & 0.000 \\
\hline
\end{tabular}

Table 6: Inverse of CC Matrix

\begin{tabular}{|c|c|c|c|c|c|c|c|c|c|c|c|c|c|c|}
\hline 89.58244 & -8.23989 & 19.0674 & -13.9619 & -69.8066 & -498.213 & 209.9039 & 111.5498 & -43.8296 & 6.033514 & 117.3796 & -267.688 & -157.164 & -12.6437 & 88.14253 \\
\hline 5844471 & 68.56985 & 1087359 & -1178226 & -6150015 & $-3.40 E+07$ & 17337558 & 9204905 & -2986336 & 1124862 & 1911533 & $-2.50 \mathrm{E}+07$ & $-1.20 \mathrm{E}+07$ & -5402420 & 6118754 \\
\hline-1527327 & -22.7285 & -375557 & 470242.1 & 1673259 & 11953464 & -7291901 & -3628974 & 900465 & -527846 & -1361864 & 9873048 & 3862733 & 3066123 & -1946844 \\
\hline 5928941 & 79.00822 & 1456552 & -1416526 & -6065459 & $-4.10 \mathrm{E}+07$ & 22828587 & 10525356 & -4133513 & 1142219 & 5740519 & $-2.90 \mathrm{E}+07$ & $-1.50 \mathrm{E}+07$ & -4622520 & 7820639 \\
\hline 53767678 & 1329.349 & -4186093 & 34800770 & $-1.30 E+08$ & $4.46 \mathrm{E}+08$ & $-6.20 \mathrm{E}+08$ & $-3.09 E+08$ & 10807450 & $-6.70 \mathrm{E}+07$ & 4.13E+08 & $8.65 E+08$ & $2.14 \mathrm{E}+08$ & $4.23 E+08$ & $-1.00 E+08$ \\
\hline $3.21 \mathrm{E}+08$ & 4827.16 & 43022542 & -425610 & $-4.20 \mathrm{E}+08$ & $-7.60 \mathrm{E}+08$ & $-1.40 E+08$ & $-9.5 \mathrm{E}+07$ & $-1.08 E+08$ & $-5.10 E+07$ & $7.52 \mathrm{E}+08$ & 56700490 & $-3.40 E+08$ & $4.48 \mathrm{E}+08$ & $2.17 \mathrm{E}+08$ \\
\hline$-4.50 E+07$ & 1056.329 & $-2.50 E+07$ & 80775691 & $-3.60 \mathrm{E}+07$ & $1.36 \mathrm{E}+09$ & $-1.30 \mathrm{E}+09$ & $-6.86 \mathrm{E}+08$ & 60000075 & $-1.40 \mathrm{E}+08$ & $5.97 \mathrm{E}+08$ & $1.85 E+09$ & $6.43 E+08$ & $8.46 \mathrm{E}+08$ & $-2.40 E+08$ \\
\hline$-2.10 \mathrm{E}+08$ & -3747.97 & $-2.00 E+07$ & $-2.80 \mathrm{E}+07$ & $3.00 E+08$ & 48211406 & $5.60 \mathrm{E}+08$ & $3.09 \mathrm{E}+08$ & 57984921 & 83903944 & $-7.40 E+08$ & $-7.00 E+08$ & 7598047 & $-6.30 E+08$ & $-6.10 E+07$ \\
\hline$-1.30 \mathrm{E}+07$ & -1533.1 & 3036311 & $-4.50 E+07$ & $6.60 \mathrm{E}+07$ & $-5.80 E+08$ & 7.16E+08 & $3.92 \mathrm{E}+08$ & 2574008 & 92788837 & $-4.90 \mathrm{E}+08$ & $-1.10 E+09$ & $-3.40 E+08$ & $-5.80 E+08$ & 93212128 \\
\hline-5658244 & -191.912 & -1828362 & -1968985 & $1.00 \mathrm{E}+07$ & 2211466 & 27550727 & 18229502 & 5818900 & 6885361 & $-4.50 E+07$ & $-5.90 \mathrm{E}+07$ & $-1.50 \mathrm{E}+07$ & $-4.00 \mathrm{E}+07$ & 5812754 \\
\hline $2.29 \mathrm{E}+08$ & 8982.95 & -3833674 & $2.07 E+08$ & $-4.70 E+08$ & $2.52 \mathrm{E}+09$ & $-3.40 E+09$ & $-1.91 \mathrm{E}+09$ & $-1.7 \mathrm{E}+07$ & $-4.40 \mathrm{E}+08$ & $2.53 E+09$ & $4.67 E+09$ & $1.21 \mathrm{E}+09$ & $2.90 \mathrm{E}+09$ & $-3.50 E+08$ \\
\hline$-1.70 \mathrm{E}+08$ & 1805.592 & 11748036 & 64757954 & $2.30 \mathrm{E}+08$ & $7.98 \mathrm{E}+08$ & $-7.30 \mathrm{E}+08$ & $-5.55 E+08$ & $-7.4 \mathrm{E}+07$ & $-1.70 E+08$ & $7.01 \mathrm{E}+08$ & $1.53 E+09$ & $7.35 E+08$ & $9.56 \mathrm{E}+08$ & $-7.70 E+07$ \\
\hline$-6.90 \mathrm{E}+08$ & -15055.6 & $-3.40 E+07$ & $-2.20 \mathrm{E}+08$ & 1.10E+09 & $-1.90 E+09$ & $4.02 E+09$ & $2.14 \mathrm{E}+09$ & $1.06 \mathrm{E}+08$ & $5.10 \mathrm{E}+08$ & $-3.50 E+09$ & $-5.20 E+09$ & $-8.80 E+08$ & $-3.50 E+09$ & 27564092 \\
\hline $4.21 \mathrm{E}+08$ & 13540.23 & 15163498 & $2.68 \mathrm{E}+08$ & $-8.60 E+08$ & $2.86 \mathrm{E}+09$ & $-4.50 E+09$ & $-2.46 E+09$ & $-8.2 E+07$ & $-5.90 E+08$ & $3.63 E+09$ & $6.42 \mathrm{E}+09$ & $1.62 \mathrm{E}+09$ & $3.84 \mathrm{E}+09$ & $-4.10 E+08$ \\
\hline $1.27 \mathrm{E}+08$ & -38912.6 & 1.10E+08 & $-1.40 E+09$ & 1.30E+09 & $-1.90 \mathrm{E}+10$ & 2.16E+10 & 1.19E+10 & $-1.61 \mathrm{E}+08$ & $2.85 E+09$ & $-1.40 \mathrm{E}+10$ & $-3.20 E+10$ & $-1.10 \mathrm{E}+10$ & $-1.70 \mathrm{E}+10$ & $3.73 E+09$ \\
\hline
\end{tabular}




\subsubsection{RZ Vector}

$\mathrm{RZ}$ vector is defined as the matrix product of the response function $₫$ on Table 3 and the pseudo variables $(\mathrm{z})$ on Table 4. The result is as presented below.

$\begin{array}{cc}\Sigma(Z 1 . F(z)) & 4.046346636 \\ \Sigma(Z 2 . F(z)) & 7.804881844 \\ \Sigma(Z 3 . F(z)) & 12.29754542 \\ \Sigma(Z 4 . F(z)) & 19.6312261 \\ \Sigma(Z 1 Z 2 . F(z)) & 0.73348255 \\ \Sigma(Z 1 Z 3 . F(z)) & 1.135114412 \\ \Sigma(Z 1 Z 4 . F(z)) & 1.800191249 \\ \Sigma(Z 2 Z 3 . F(z)) & 2.187311458 \\ \Sigma(Z 2 Z 4 . F(z)) & 3.4514716 \\ \Sigma(Z 3 Z 4 . F(z)) & 5.5117773\end{array}$

$\begin{array}{cc}\Sigma(Z 1 Z 2 Z 3 . F(z)) & 0.20515741 \\ \Sigma(Z 1 Z 2 Z 4 . F(z)) & 0.32163503 \\ \Sigma(Z 1 Z 3 Z 4 . F(z)) & 0.50492043 \\ \Sigma(Z 2 Z 3 Z 4 . F(z)) & 0.9673951 \\ \Sigma(Z 1 Z 2 Z 3 Z 4 . F(z)) & 0.08997551\end{array}$

\subsubsection{Coefficients of the Reegression Equation}

The coefficients of the regression equation given in Equation 1 can be obtained using Equation 6. That is, the coefficients of the regression equation are the matrix product of the $\mathrm{RZ}$ vector and the inverse of the $\mathrm{CC}$ matrix given on Table 6 . The result is as given in Table 7 .

Table 7: Coefficients of the Regression Equation

\begin{tabular}{|c|c|c|c|c|c|c|c|c|c|c|c|c|c|c|}
\hline \multicolumn{15}{|c|}{ Table 7: Coefficients of the Regression Equation } \\
\hline$\alpha 1$ & a2 & a3 & a4 & a12 & a13 & a14 & a23 & $\alpha 24$ & a34 & a123 & a124 & a134 & a234 & a1234 \\
\hline 0 & -143.86 & 113.11 & -50.69 & 20681.08 & 23469.28 & 40321.73 & -34149.52 & -30668.19 & -2187.68 & 148746.68 & 58404.68 & 175403.73 & 199524.86 & -829819.01 \\
\hline
\end{tabular}

Substituting the coefficients in Table 7 into Equation 1 will give the regression model for the prediction of $28^{\text {th }}$ day flexural strengths of periwinkle shell-river gravel concrete given in Equation 8.
$\mathrm{F}(\mathrm{z})$ $=$

$50.68 z_{4}+20681.08 z_{1} z_{2}+23469.28 z_{1} z_{3}+40321.73 z_{1} z_{4}-$

$34149.52 \mathrm{z}_{2} \mathrm{z}_{3}-30668.19 \mathrm{z}_{2} \mathrm{z}_{4}-$

$2187.68 z_{3} z_{4}+148746.68 z_{1} z_{2} z_{3}+58404.68 z_{1} z_{2} z_{4}-$

$175403.73 z_{1} z_{3} z_{4}+199524.86 z_{2} z_{3} z_{4}-829819.01 z_{1} z_{2} z_{3} z_{4}$
(8)

The flexural strengths from the formulated model given in Table 8 are obtained through substitution of the pseudo variables in Table 4 into Equation 8. These values from the model are placed side by side with that from the laboratory experiment for easy comparison and statistical analysis as represented on Table 9.

Table 8: Laboratory and Model Flexural Strengths of Periwinkle shell-sand stone concrete

\section{ACTUAL MIX RATIOS}

\begin{tabular}{|l|l|l|l|l|l|l|l|l|l|l|l|l|l|l|r|r|}
\hline MIX NO & & $\mathrm{N} 1$ & $\mathrm{~N} 2$ & $\mathrm{~N} 3$ & $\mathrm{~N} 4$ & $\mathrm{~N} 12$ & $\mathrm{~N} 13$ & $\mathrm{~N} 14$ & $\mathrm{~N} 23$ & $\mathrm{~N} 24$ & $\mathrm{~N} 34$ & $\mathrm{~N} 123$ & $\mathrm{~N} 124$ & $\mathrm{~N} 134$ & $\mathrm{~N} 234$ & $\mathrm{~N} 1234$ \\
\hline $\begin{array}{l}\text { AVERAGE } \\
\begin{array}{l}\text { FLEXURAL } \\
\text { STRENGTH } \\
\left(\mathrm{N} / \mathrm{mm}^{2}\right)\end{array}\end{array}$ & YMODEL & 2.95 & 2.32 & 2.41 & 3.27 & 1.83 & 3.89 & 1.62 & 2.99 & 2.75 & 3.00 & 3.48 & 4.42 & 2.26 & 2.72 & 3.88 \\
\cline { 2 - 13 } & 2.63 & 2.69 & 2.49 & 3.13 & 3.12 & 3.29 & 2.40 & 2.77 & 2.67 & 3.04 & 3.17 & 2.65 & 3.54 & 3.04 & 3.15 \\
\hline
\end{tabular}

\section{CONTROL MIX RATIOS}

\begin{tabular}{|l|l|l|l|l|l|l|l|l|l|l|l|l|l|l|l|l|}
\hline MIX NO & & $\mathrm{C} 1$ & $\mathrm{C} 2$ & $\mathrm{C} 3$ & $\mathrm{C} 4$ & $\mathrm{C} 5$ & $\mathrm{C} 6$ & $\mathrm{C} 7$ & $\mathrm{C} 8$ & $\mathrm{C} 9$ & $\mathrm{C} 10$ & $\mathrm{C} 11$ & $\mathrm{C} 12$ & $\mathrm{C} 13$ & $\mathrm{C} 14$ & $\mathrm{C} 15$ \\
\hline $\begin{array}{l}\text { AVERAGE } \\
\begin{array}{l}\text { FLEXURAL } \\
\text { STRENGTH } \\
\left(\mathrm{N} / \mathrm{mm}^{2}\right)\end{array}\end{array}$ & YMODEL & 3.34 & 3.15 & 2.33 & 3.08 & 3.37 & 2.57 & 2.88 & 2.35 & 3.55 & 2.98 & 1.98 & 3.15 & 3.13 & 2.15 & 3.37 \\
\cline { 2 - 22 } & 2.52 & 2.45 & 2.52 & 2.85 & 2.77 & 3.05 & 2.97 & 2.75 & 2.87 & 2.94 & 2.34 & 1.90 & 2.67 & 2.79 & 2.58 \\
\hline
\end{tabular}

\subsection{Fisher Test for Adequacy of Model}

$\underline{\text { LEGEND }}$

$\mathrm{Y}_{\mathrm{E}}=$ response from experiment

$\mathrm{Y}_{\mathrm{M}}=$ response from model

$\tilde{Y}_{E}=$ mean response from experiment $=\sum Y_{E} / N$

$\tilde{Y}_{\mathrm{M}}=$ mean response from model $=\sum \mathrm{Y}_{\mathrm{M}} / \mathrm{N}$

$\mathrm{N}=$ total points of observation (for controls) $=15$
$\mathrm{S}_{\mathrm{E}}^{2}=\sum\left(\mathrm{Y}_{\mathrm{E}}-\tilde{\mathrm{Y}}_{\mathrm{E}}\right)^{2} /(\mathrm{N}-1)$

$\mathrm{S}_{\mathrm{M}}{ }^{2}=\sum\left(\mathrm{Y}_{\mathrm{M}}-\tilde{\mathrm{Y}}_{\mathrm{M}}\right)^{2} /(\mathrm{N}-1)$

$(\mathrm{N}-1)=$ degree of freedom $=15-1=14$

$\mathrm{S}_{\mathrm{E}}^{2}=$ variance from the experiment

$\mathrm{S}_{\mathrm{M}}{ }^{2}=$ variance from the model 
Table 9: Periwinkle Shell-Sand Stone Concrete

\begin{tabular}{|l|l|l|l|l|l|l|}
\hline RESPONSE & & & & & & \\
\hline C1 & 2.52 & 3.34 & -0.14 & 0.45 & 0.0196 & 0.2025 \\
\hline C2 & 2.45 & 3.15 & -0.21 & 0.26 & 0.0441 & 0.0676 \\
\hline C3 & 2.52 & 2.33 & -0.14 & -0.56 & 0.0196 & 0.3136 \\
\hline C4 & 2.85 & 3.08 & 0.19 & 0.19 & 0.0361 & 0.0361 \\
\hline C5 & 2.77 & 3.37 & 0.11 & 0.48 & 0.0121 & 0.2304 \\
\hline C6 & 3.05 & 2.57 & 0.39 & -0.32 & 0.1521 & 0.1024 \\
\hline C7 & 2.97 & 2.88 & 0.31 & -0.01 & 0.0961 & 0.0001 \\
\hline C8 & 2.75 & 2.35 & 0.09 & -0.54 & 0.0081 & 0.2916 \\
\hline C9 & 2.87 & 3.55 & 0.21 & 0.66 & 0.0441 & 0.4356 \\
\hline C10 & 2.94 & 2.98 & 0.28 & 0.09 & 0.0784 & 0.0081 \\
\hline C11 & 2.34 & 1.98 & -0.32 & -0.91 & 0.1024 & 0.8281 \\
\hline C12 & 1.90 & 3.15 & -0.76 & 0.26 & 0.5776 & 0.0676 \\
\hline C13 & 2.67 & 3.13 & 0.01 & 0.24 & 0.0001 & 0.0576 \\
\hline C14 & 2.79 & 2.15 & 0.13 & -0.74 & 0.0169 & 0.5476 \\
\hline C15 & 2.58 & 3.37 & -0.08 & 0.46 & 0.0064 & 0.2304 \\
\hline & $\sum \mathrm{Y}_{\mathrm{E}}=$ & $\sum \mathrm{Y}_{\mathrm{M}}=$ & & & $\sum\left(\mathrm{Y}_{\mathrm{E}^{-}} \tilde{\mathrm{Y}}_{\mathrm{E}}\right)^{2}=$ & $\sum\left(\mathrm{Y}_{\mathrm{M}^{-}} \tilde{\mathrm{Y}}_{\mathrm{M}}\right)^{2}=$ \\
\hline
\end{tabular}

$\tilde{\mathrm{Y}}_{\mathrm{E}}=\sum \mathrm{Y}_{\mathrm{E}} / \mathrm{N}=39.97 / 15=2.66$

$\tilde{\mathrm{Y}}_{\mathrm{M}}=\sum \mathrm{Y}_{\mathrm{M}} / \mathrm{N}=43.38 / 15=2.89$

$\mathrm{S}_{\mathrm{E}}{ }^{2}=\sum\left(\mathrm{Y}_{\mathrm{E}^{-}} \tilde{\mathrm{Y}}_{\mathrm{E}}\right)^{2} /(\mathrm{N}-1)=1.2137 / 14=0.0867$

$\mathrm{S}_{\mathrm{M}}{ }^{2}=\sum\left(\mathrm{Y}_{\mathrm{M}^{-}} \tilde{\mathrm{Y}}_{\mathrm{M}}\right)^{2} /(\mathrm{N}-1)=2.5912 / 14=0.1851$ Therefore,

$\mathrm{S}_{1}^{2}=0.1851$ and $\mathrm{S}_{2}^{2}=0.0867$

$\mathrm{F}_{\text {calculated }}=\mathrm{S}_{1}{ }^{2} / \mathrm{S}_{2}{ }^{2}=0.1851 / 0.0867=2.13$

$\mathrm{F}_{\text {table }}=\mathrm{F}_{0.05}(14,14)$

From appendix B, $\mathrm{F}_{0.05}(14,14)=2.48$

$1 / \mathrm{F}_{\text {table }}=0.403$

Therefore, $0.403<2.13<2.48$

Thus, the condition $1 / \mathrm{F}<\mathrm{S}_{1}{ }^{2} / \mathrm{S}_{2}{ }^{2}<\mathrm{F}$ has been satisfied.

Null hypothesis will be accepted.

\section{CONCLUSION}

From the statistical analysis carried out on the generated regression model for the prediction of flexural strengths of concrete made with periwinkle shells and sand stone, blended as a single component at a volumetric mix ratio of $1: 1$, the calculated f-value of 2.13 was less than the allowable f-value of 2.48 from the statistical table at $5 \%$ significance level. This means that the flexural strengths results from the experiment and those from the model compare favourably with each other. It can therefore be concluded that the regression model proved adequate for the prediction of $28^{\text {th }}$-day flexural strengths of concrete made from equal volume of sand stone and periwinkle shells at $95 \%$ confidence level or 5\% significance level. This model is recommended for use in concrete industry or construction industry for easy forecast of flexural strengths of lightweight concretes whose mix ratios are within the boundaries provided in this research work.

\section{REFERENCES}

[1] Adewuyi, A. P., \&Adegoke, T. (2009). Exploratory Study of Periwinkle Shells As Coarse Aggregates In Concrete Works. ARPN Journal of Engineering and Applied Science, 3(6), 1-5.

[2] Amaziah, W. O., Idongesit, U. F., \& Theodore, A. I. (2013). Exploratory Study of Crushed Periwinkle Shell as Partial Replacement For Fine Aggregate In Concrete. Journal of Emerging Trends in Engineering and Applied Sciences, 4(6), 823-827.

[3] Awe, F. C., Aribisala, J. O., \&Ogundipe, O. M. (2014). Investigation Into The Suitability of Periwinkle Shell as Filler Material. Journal of Emerging Trends In Engineering and Applied Sciences, 5(4), 237-242.

[4] BS EN 197. (2000). Specification for Ordinary and Rapid-Hardening Portland Cement. part 1, London: British Standard Institution.

[5] Elijah, I. O., Sunda, B., \&Dorcas, D. S. B. (2009). Uses of Seas Shells For Civil Construction Works In Coastal Bayelsa State, Nigeria: A Waste Management Perspective. Research Journal Of Biological Sciences, 4(9), 1025-1031.

[6] Ettu, L. O., Ibearugbulem, O. M., Ezeh, J. C., \& Anya, U. C. (2013). A Reinvestigation of the Prospects of Using Periwinkle Shell as Partial Replacement for Granite in Concrete. International Journal of Engineering Science Invention, 2(3), 54-59.

[7] Falade, F., Ikponmwosa, E. E., \&Ojedira, N. I. (2010). Behaviour of Lightweight Concrete Containing Periwinkle Shells at Elevated Temperature. Journal of Engineering Science And Technology, 5(4), 379-390.

[8] Ibearugbulem, O. M., Ettu, L.O., Ezeh, J. C., \& Anya, U. C. (2013). A New Regression Model For Optimising Concrete Mixes. International Journal of Engineering Science And Research Technology, 2(7), 1735-1742.

[9] Njoku, R. E., Okon, A. E., \&Ikpali, T. C. (2011). Effects Of Variation of Particle Size And Weight Fraction On The Tensile Strength And Modulus of Periwinkle Shell Reinforced Polyester Composite. Nigerian Journal of Technology, 30(2), 87-93.

[10] Ohimain, E. I., Bassey, S., \&Bawo, D. D. S. (2009). Uses of Sea Shells For Civil Construction Works In Coastal Bayelsa State, Nigeria: A Waste Management Perspective. Medwell Journal: Scientific Research Publishing Company, 4(9), 1025-1031. 
[11] Osadebe, N. N. (2003). Generalised mathematical modelling of Compressive strength of Normal Concrete as a Multivariant Function of the Properties of its Constituent Components. A Paper Delivered at College of Engineering University of Nigeria, Nsukka, Unpublished.

[12] Osarenwinda, J. O., \&Awaro, A. O. (2009). The Potential Use of Periwinkle Shell As Coarse Aggregate For Concrete. Advanced Material Research, 62, 39-43.

[13] Scheffe, H. (1950). Experiment with mixtures.Journal of Royal Statistics Society. Series B(20): $344-360$.

\section{BIOGRAPHIES}

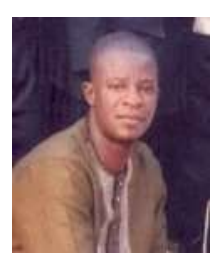

Engr. Dr. Ibearugbulem Mathias Owus

Engr. Dr. Ibearugbulem M.O is a senior lecturer in department of civil engineering, federal university of technology owerri. He obtained his Ph.D in structural engineering at federal university of technology owerri, Imo state, Nigeria. His research area is in dry/analytical structures and concrete production. He is a corporate member of Nigerian Society of Engineers and Council for the Regulation of Engineering in Nigeria.

Email: ibeowums@yahoo.com

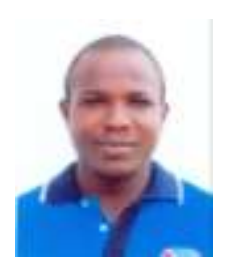

\section{Ajoku, Chinedu Azubuike}

Mr Ajoku C.A is an assistant lecturer in the department of civil engineering, federal university of technology owerri. $\mathrm{He}$ obtained his M.Eng and B.Eng in structural engineering from federal university of technology owerri, Imo state, Nigeria. His area of research is in the conversion of industrial and agricultural waste materials into concrete materials for the production of affordable housing unit.

Email: ajokuchineduazubuike@yahoo.com

\section{Iwuoha, Stanley Emeka}

Mr Iwuoha is a graduate assistant in the department of civil engineering, federal university of technology owerri. He is currently running a masters degree programme in structural engineering. His area of research is in dry/ analytical structures.

\section{Iwuohastanley1@gmail.com}

